\title{
KRYLOV-BOGOLIUBOV-MITROPOLSKY AVERAGING USED TO CONSTRUCT EFFECTIVE HAMILTONIANS IN THE THEORY OF STRONGLY CORRELATED ELECTRON SYSTEMS
}

\author{
A. P. Saiko*
}

\begin{abstract}
We show that the Krylov-Bogoliubov-Mitropolsky averaging in the canonical formulation can be used as a method for constructing effective Hamiltonians in the theory of strongly correlated electron systems. As an example, we consider the transition from the Hamiltonians of the Hubbard and Anderson models to the respective Hamiltonians of the t-J and Kondo models. This is a very general method, has several advantages over other methods, and can be used to solve a wide range of problems in the physics of correlated systems.
\end{abstract}

PACS: 71.10.Fd, 71.27.+a, 75.30.Mb

Model Hamiltonians used to describe strongly correlated electron systems with the electron potential energy much greater than its kinetic energy can be significantly simplified by reducing them to effective Hamiltonians in spin variables. Such a simplification can be realized by the initial Hamiltonian renormal-izations aimed at eliminating high-energy states and at passing to a subspace with lower energies of the quantum states. Eliminating high-energy states is justified in this case because the system properties (e.g., electrical conduction, magnetization, etc.) under the usual laboratory conditions are determined by the ground state and the low-energy excitations. As a rule, the effective Hamiltonians are constructed using the canonical transformation method [1], [2], which allows eliminating an "off-diagonal"small perturbation operator, which is responsible for transitions

*saiko@ifttp.bas-net.by 
between low- and high-energy states, from the original Hamiltonian. Sometimes, the perturbation theory is also used in systems with degenerate states [3]. Here, we propose a method for constructing effective Hamiltonians based on using the Krylov-Bogoliubov-Mitropolsky (KBM) averaging method [4] developed for solving problems in the theory of nonlinear oscillations. The averaging method allows eliminating the rapidly oscillating terms (the high-energy states) in any perturbation order in the Hamiltonian written in the interaction representation, which leads to an effective Hamiltonian written in the approximately "diagonal" form. In what follows, as examples, we show how the KBM averaging method in its canonical formulation can be used to obtain the t-J model Hamiltonian from the Hubbard Hamiltonian and the Kondo model Hamiltonian from the Anderson model Hamiltonian.

The paradigmatic model in the theory of strongly correlated electron systems is the Hubbard model. The Hubbard Hamiltonian is written as [1], [2]:

$$
H=-t \sum_{<i j>, \sigma}\left(c_{i \sigma}^{+} c_{j \sigma}+H . c .\right)+U \sum_{i} n_{i \uparrow} n_{i \downarrow} \equiv H_{t}+H_{U}
$$

where $H_{t}$ is the kinetic (band) term describing the motion over the lattice sites, $H_{U}$ is the operator of the Coulomb energy of repulsion of two electrons at the same site; $c_{i \sigma}^{+}$is the operator of creation of an electron with the spin $\sigma$ at the site $i$, and $t$ is the matrix element of electron transition from a given site to its neighbor. In the case of large Coulomb energy $U$, the appearance of two electrons at the same site is energetically unfavorable, and the original band splits into two Hubbard subbands: the upper and the lower (with a gap between them) corresponding to the one-electron and two-electron states. In the case of a half-filled band (one electron at the site, $n=\sum_{\sigma} n_{\sigma}=1$ ), the Mott transition occurs for $U \sim t$, i.e., the dielectric ground state appears, and an indirect exchange coupling of antiferromagnetic type is established between the electrons at the site. The so-called t-J model is thus realized, which describes the propagation of holes in the lower subband against the background of interacting spins for $n<1$. If the t-J model is derived from the Hubbard model, then the Coulomb term is usually taken as the zeroth-order approximation, and the kinetic term is considered a perturbation. In the interaction representation, where $H_{U}$ is taken as the zeroth-order Hamiltonian, the kinetic term is divided into 
parts describing the energetically more favorable processes (in the interior of the Hubbard subbands) and the less favorable processes (between the Hubbard subbands). Indeed, in this representation,

$$
c_{j \sigma}(\tau)=e^{i H_{U} \tau} c_{j \sigma} e^{-i H_{U} \tau}=c_{j \sigma}\left(1-n_{j \bar{\sigma}}\right)+e^{-i U \tau} c_{j \sigma} n_{j \bar{\sigma}}
$$

where $\bar{\sigma}=-\sigma$ and the kinetic term $H_{t}(\tau)=e^{i H_{U} \tau} H_{t} e^{-i H_{U} \tau}$ has the manifestly multiparticle character:

$$
\begin{gathered}
H_{t}(\tau)=-t \sum_{<i j>, \sigma}\left[\left(1-n_{i \bar{\sigma}}\right) c_{i \sigma}^{+} c_{j \sigma}\left(1-n_{j \bar{\sigma}}\right)+n_{i \bar{\sigma}} c_{i \sigma}^{+} c_{j \sigma} n_{j \bar{\sigma}}+H . c .\right]- \\
-t \sum_{<i j>, \sigma}\left[n_{i \bar{\sigma}} c_{i \sigma}^{+} c_{j \sigma}\left(1-n_{j \bar{\sigma}}\right)+n_{j \bar{\sigma}} c_{j \sigma}^{+} c_{i \sigma}\left(1-n_{i \bar{\sigma}}\right)\right] e^{i U \tau}- \\
-t \sum_{<i j>, \sigma}\left[\left(1-n_{i \bar{\sigma}}\right) c_{i \sigma}^{+} c_{j \sigma} n_{j \bar{\sigma}}+\left(1-n_{j \bar{\sigma}}\right) c_{j \sigma}^{+} c_{i \sigma} n_{i \bar{\sigma}}\right] e^{-i U \tau} \equiv \\
\equiv H_{t}^{0}+H_{t}^{+}(\tau)+H_{t}^{-}(\tau) .
\end{gathered}
$$

The first and second terms in $H_{t}^{0}$ are responsible for the electron kinetics in the respective lower and upper Hubbard subbands; $H_{t}^{+}(\tau)$ describes the appearance of the second electron at a site already containing an electron, i.e., the transition from a low-energy state to a high-energy state (from the lower Hubbard subband into the upper); and $H_{t}^{-}(\tau)$ represents the reverse process in which the number of doubly occupied sites (sites with a pair of electrons) decreases by unity. The existence of the rapidly oscillating factors $e^{ \pm i U \tau}$ in $H_{t}^{+}(\tau)$ and $H_{t}^{-}(\tau)$ additionally indicates that the processes with transitions between the Hubbard subbands are unfavorable.

The KBM averaging method allows eliminating the rapidly oscillating "off-diagonal" terms $H_{t}^{ \pm}(\tau)$ in any perturbation order in $t$ (more precisely, in $t / U$ ). and can be applied to Hamiltonian (2). We briefly describe the method in the canonical formalism [4]-[6].

We consider the Liouville equation for the density matrix of a quantum system

$$
\frac{\partial \rho}{\partial \tau}=-i\left[H_{0}+H_{1}, \rho\right] \equiv-i\left(L_{0}+L_{1}\right) \rho
$$

where $H_{0}$ is the unperturbed "diagonal" Hamiltonian of the system, $H_{1}$ is a small perturbation, i.e., the "off-diagonal term," and $L_{0}$ and $L_{1}$ are the Liouvillians corresponding to the Hamiltonians. It follows from the condition $\left\|H_{0}\right\| \gg\left\|H_{1}\right\|$ where $\|\ldots\|$ denotes the value of an operator in frequency units, that the fast motion with the period $2 \pi /\left\|H_{1}\right\|$ is superimposed on a slower process characterized 
by time of the order of $\sim\left\|H_{1}\right\|^{-1}$. B (3) We can pass to the interaction representation $\sigma=e^{i L_{0} t} \rho$, $L_{1}(\tau)=e^{i L_{0} \tau} L_{1} e^{-i L_{0} \tau}$ in Eq. (3) and then apply the KBM averaging method to the equation

$$
\frac{\partial \sigma}{\partial \tau}=-i L_{1}(\tau) \sigma
$$

in order to eliminate the rapidly oscillating terms and to construct an approximately "diagonal" effective Hamiltonian (Liouvillian). We briefly describe this procedure in the simplest form.

We note that because the function $L_{1}(\tau)$, is periodic, it can be expanded in the Fourier series

$$
L_{1}(\tau)=\sum_{n} L_{1}^{(n)} e^{i \omega_{n} \tau}, \quad L_{1}^{(n)}=\frac{1}{T} \int_{0}^{T} d \tau L_{1}(\tau) e^{-i \omega_{n} \tau},
$$

where $\omega_{n}=2 \pi n / T$ and $T$ is the period, which, in particular, can coincide with the period $2 \pi /\left\|H_{0}\right\|$ or be multiple of it. Because we consider not the fast time "vibration" of the density matrix but its slow evolution, i.e., the motion averaged over several time periods $T$, it is natural to define the averaging operation

$$
P^{\tau} \sigma(\tau)=\frac{1}{T} \int_{0}^{T} d \tau \sigma(\tau) \equiv\langle\sigma(\tau)\rangle,
$$

where the projection operator $P^{\tau}$ is the operator of averaging the rapidly varying quantities. We also define $Q^{\tau}=1-P^{\tau}$ (it is easy to see that $P^{\tau} P^{\tau}=P^{\tau}, Q^{\tau} Q^{\tau}=Q^{\tau}$, and $P^{\tau} Q^{\tau}=Q^{\tau} P^{\tau}=0$ ), We can then write

$$
\sigma(\tau)=P^{\tau} \sigma(\tau)+Q^{\tau} \sigma(\tau)
$$

i.e., we decompose the real motion of the system described by the density matrix $\sigma(\tau)$ into the averaged $P^{\tau} \sigma$ and fast "vibration" $Q^{\tau} \sigma$. Substituting expression (7) in (4) and acting on it from the left successively by the operators $P^{\tau}$ and $Q^{\tau}$ we obtain the two coupled equations

$$
\begin{aligned}
& \frac{\partial}{\partial \tau}\left(P^{\tau} \sigma\right)=-i P^{\tau} L_{1}(\tau) P^{\tau} \sigma-i P^{\tau} L_{1}(\tau) Q^{\tau} \sigma \\
& \frac{\partial}{\partial \tau}\left(Q^{\tau} \sigma\right)=-i Q^{\tau} L_{1}(\tau) Q^{\tau} \sigma-i Q^{\tau} L_{1}(\tau) P^{\tau} \sigma
\end{aligned}
$$

Equations (8) and (9) were derived using the periodicity property of the function $Q^{\tau} \sigma$ and the fact that $P^{\tau} \sigma$ is a slow function of time. The solution of Eq. (9) can be represented formally as

$$
Q^{\tau} \sigma=-i \int^{\tau} d \tau^{\prime} Q^{\tau^{\prime}} L_{1}\left(\tau^{\prime}\right) P^{\tau^{\prime}} \sigma\left(\tau^{\prime}\right)-i \int^{\tau} d \tau^{\prime} Q^{\tau^{\prime}} L_{1}\left(\tau^{\prime}\right) Q^{\tau^{\prime}} \sigma\left(\tau^{\prime}\right)
$$


and the operator constant in this expression is assumed to be zero: we here use the freedom to choose this constant arbitrarily because one first-order differential equation (4) was divided into two equations, Eqs. (8) and (9), at the preceding stage. We iterate expression (10) and obtain the power expansion in $L_{1}$ :

$$
Q^{\tau} \sigma \approx\left\{-i \int^{\tau} d \tau^{\prime} Q^{\tau^{\prime}} L_{1}\left(\tau^{\prime}\right)-i \int^{\tau} d \tau^{\prime} \int^{\tau^{\prime}} d \tau^{\prime \prime} Q^{\tau^{\prime}} L_{1}\left(\tau^{\prime}\right) Q^{\tau^{\prime \prime}} L_{1}\left(\tau^{\prime \prime}\right) \ldots,\right\}\langle\sigma\rangle
$$

where the slowly varying function $P^{\tau} \sigma \equiv\langle\sigma\rangle$ is brought outside the integrand. Substituting formula (11) in (8), we obtain an equation for slowly varying quantities. For example, in the second order in $L_{1}$, we have

$$
\frac{\partial}{\partial \tau}\langle\sigma\rangle=-i\left\langle L_{1}(\tau)\right\rangle\langle\sigma\rangle-\left\langle\int^{\tau} d \tau^{\prime}\left\{L_{1}\left(\tau^{\prime}\right)\left(L_{1}\left(\tau^{\prime}\right)-\left\langle L_{1}\left(\tau^{\prime}\right)\right\rangle\right)\right\}\right\rangle\langle\sigma\rangle \equiv-i L_{e f f}\langle\sigma\rangle,
$$

where we introduce the effective Liouvillian

$$
L_{e f f}=\left\langle L_{1}(\tau)\right\rangle-i\left\langle\int^{\tau} d \tau^{\prime}\left\{L_{1}\left(\tau^{\prime}\right)\left(L_{1}\left(\tau^{\prime}\right)-\left\langle L_{1}\left(\tau^{\prime}\right)\right\rangle\right)\right\}\right\rangle
$$

which is associated with the effective Hamiltonian

$$
H_{e f f}=\left\langle H_{1}(\tau)\right\rangle+\frac{i}{2}\left\langle\left[\int^{\tau} d \tau^{\prime}\left(H_{1}\left(\tau^{\prime}\right)-\left\langle H_{1}\left(\tau^{\prime}\right)\right\rangle\right), H_{1}(\tau)\right]\right\rangle
$$

To derive expression (14) from (13), it is most convenient to use Fourier expansion (5).

In the Hubbard model considered here, averaging the Hamiltonian $H_{t}(\tau)$ over the period $2 \pi / U$ results in the relation $\left\langle H_{t}(\tau)\right\rangle=H_{t}^{0}$ because the averages of $H_{t}^{ \pm}(\tau)$ are zero because of the factors $e^{ \pm i U \tau}$. We use the total KBM averaging procedure, i.e., formulas (14), to obtain

$$
\begin{gathered}
H_{\text {eff }}^{(2)}=H_{t}^{0}+\frac{1}{U}\left\langle\left[H_{t}^{+}(\tau), H_{t}^{-}(\tau)\right]\right\rangle+\frac{1}{2 U}\left\langle\left[H_{t}^{+}(\tau)-H_{t}^{-}(\tau), H_{t}^{0}\right]\right\rangle= \\
=H_{t}^{0}+\frac{1}{U}\left[H_{t}^{+}(0), H_{t}^{-}(0)\right] .
\end{gathered}
$$

We note that the canonical transformation method applied to the Hubbard Hamiltonian leads to a similar effective Hamiltonian but with additional "off-diagonal" terms $\sim\left[H_{t}^{ \pm}(0), H_{t}^{0}\right]$ (see, e.g., [1], [2]), which can be neglected because they take the interband transitions into account only in the second order in $t / U[2]$ but not in the first order. In the KBM method, such terms do not appear, because the rapidly oscillating term $\left[H_{t}^{+}(\tau)-H_{t}^{-}(\tau), H_{t}^{0}\right]$ in $(15)$ is zero after averaging over the period 
$2 \pi / U$. Further, to obtain the desired result, it is necessary to substitute $H_{t}^{0}$ given in (2) in expression (15) and to perform rather simple transformations, which are described in detail in [1]. Namely, it is necessary to commute the operators, to omit the three-site terms [1], [2], and to project the obtained Hamiltonian on the lower Hubbard subband, i.e., to omit the terms multiplied on the left and on the right by the operators $n_{i \bar{\sigma}}$ and $n_{j \bar{\sigma}}$ such as, for example, the second term in $H_{t}^{0}(2): n_{i \bar{\sigma}} c_{i \sigma}^{+} c_{j \sigma} n_{j \bar{\sigma}}$ (the high-energy motion of electrons in the upper Hubbard subband is described by similar terms). Ihe obtained effective Hamiltonian $H_{e f f}^{(2)}$ is just the Hamiltonian of the t-J model:

$$
H_{e f f}^{(2)} \rightarrow H_{t-J}=-t \sum_{i, j, \sigma}\left[\left(1-n_{i \bar{\sigma}}\right) c_{i \sigma}^{+} c_{j \sigma}\left(1-n_{j \bar{\sigma}}\right)+h . c .\right]+J \sum_{i j}\left(\vec{S}_{i} \vec{S}_{j}-\frac{1}{4} n_{i} n_{j}\right),
$$

where

$$
\vec{S}_{i}=\frac{1}{2} \sum_{\sigma \sigma^{\prime}} c_{i \sigma}^{+} \vec{\tau}_{\sigma \sigma^{\prime}} c_{i \sigma^{\prime}}
$$

$\vec{\tau}$ is a vector composed of Pauli matrices, $n_{i}=\sum_{\sigma} n_{i \sigma}$ and $J=4 t^{2} / U$.

The algorithm described above for constructing an effective Hamiltonian can also be used to transform the Hamiltonian of the Anderson model into the Hamiltonian of the Kondo model. Transitions from the Anderson model to the Kondo model are usually performed using the SchriefferWolff transformation [7], [8], i.e., the canonical transformation with an anti-Hermitian operator whose form must be chosen.

The KBM averaging method also has some obvious advantages in this case. The original Anderson Hamiltonian can be written as [7], [8]

$$
\begin{gathered}
H=H_{0}+V \\
H_{0}=\sum_{k, \sigma} \varepsilon_{k} c_{k \sigma}^{+} c_{k \sigma}+\varepsilon \sum_{\sigma} c_{d \sigma}^{+} c_{d \sigma}+U c_{d \uparrow}^{+} c_{d \uparrow} c_{d \downarrow}^{+} c_{d \downarrow}, \\
V=\sum_{k, \sigma}\left(V_{k d} c_{k \sigma}^{+} c_{d \sigma}+\text { h.c. }\right)
\end{gathered}
$$

where $c_{k \sigma}^{+}$and $\varepsilon_{k}$ are the creation operator and the energy of an electron with the momentum $k$ and $\operatorname{spin} \sigma$ in the conduction band, $c_{d \sigma}^{+}$and $\varepsilon$ are the creation operator and the energy of a localized electron of the impurity atom, and $U$ is the Coulomb interaction energy between two electrons occupying the 
impurity atom. Here, $V$ describes the coupling that mixes the impurity states with band states, and $V_{k d}$ is the coupling constant.

To pass to the interaction representation $V(\tau)=e^{i H_{0} \tau} V e^{-i H_{0} \tau}$, we first write $c_{k \sigma}(\tau)=$ $e^{i H_{0} \tau} c_{k \sigma} e^{-i H_{0} \tau}$ and $c_{d \sigma}(\tau)=e^{i H_{0} \tau} c_{d \sigma} e^{-i H_{0} \tau}$ as

$$
c_{k \sigma}(\tau)=c_{k \sigma} e^{-i \varepsilon_{k} \tau}, \quad c_{d \sigma}(\tau)=c_{d \sigma}\left(1-n_{d \bar{\sigma}}\right) e^{-i \varepsilon \tau}+c_{d \sigma} n_{d \bar{\sigma}} e^{-i(\varepsilon+U) \tau}
$$

As a result, we obtain

$$
V(\tau)=\sum_{k, \sigma}\left\{V_{k d}\left[e^{i\left(\varepsilon_{k}-\varepsilon\right) \tau} c_{k \sigma}^{+} c_{d \sigma}\left(1-n_{d \bar{\sigma}}\right)+e^{i\left(\varepsilon_{k}-\varepsilon-U\right) \tau} c_{k \sigma}^{+} c_{d \sigma} n_{d \bar{\sigma}}\right]+H . c .\right\}
$$

We take all energies relative to the Fermi level. Then, in the temperature region under study, the energies $\varepsilon_{k}$ (or $\varepsilon_{k}-\mu$ instead of $\varepsilon_{k}$, where $\mu$ is the Fermi energy) are small, $\varepsilon$ is negative, and $U$, $|\varepsilon| \gg \varepsilon_{k}$.

The time average of the Hamiltonian $V(\tau)$ is zero in the first-order of the perturbation theory because of fast oscillations of the factors $e^{ \pm i\left(\varepsilon_{k}-\varepsilon\right) \tau}$ and $e^{ \pm i\left(\varepsilon_{k}-\varepsilon-U\right) \tau}$. In the second order, the KBM average of (14) is

$$
\begin{gathered}
H_{e f f}^{(2)}(\tau)=\frac{i}{2}\left\langle\left[\int^{\tau} d \tau^{\prime} V\left(\tau^{\prime}\right), V(\tau)\right]\right\rangle= \\
=-\frac{1}{2} \sum_{k, k^{\prime}, \sigma}\left[f\left(k, k^{\prime}, U\right)-f\left(k, k^{\prime}, 0\right)\right]\left(c_{k \sigma}^{+} c_{k^{\prime} \bar{\sigma}} c_{d \bar{\sigma}}^{+} c_{d \sigma}-c_{k \sigma}^{+} c_{k^{\prime} \sigma} c_{d \bar{\sigma}}^{+} c_{d \bar{\sigma}}\right) e^{i\left(\varepsilon_{k}-\varepsilon_{k^{\prime}}\right) \tau}+ \\
+\frac{1}{2} \sum_{k, k^{\prime}, \sigma} f\left(k, k^{\prime}, 0\right) c_{k \sigma}^{+} c_{k^{\prime} \sigma} e^{i\left(\varepsilon_{k}-\varepsilon_{k^{\prime}}\right) \tau}- \\
-\frac{1}{2} \sum_{k, \sigma} f(k, k, 0) n_{d \sigma}-\frac{1}{2} \sum_{k, \sigma}[f(k, k, U)-f(k, k, 0)] n_{d \sigma} n_{d \bar{\sigma}}
\end{gathered}
$$

where

$$
f\left(k, k^{\prime}, U\right)=V_{k d} V_{k^{\prime} d}^{*}\left(\frac{1}{\varepsilon_{k}-\varepsilon-U}+\frac{1}{\varepsilon_{k^{\prime}}-\varepsilon-U}\right),
$$

and $e^{i\left(\varepsilon_{k}-\varepsilon_{k^{\prime}}\right) \tau}$ are slowly varying factors. Passing to the Heisenberg representation in expression (22), we obtain the effective Hamiltonian

$$
H_{\text {eff }}^{(2)}(\tau) \rightarrow H_{e f f, H}^{(2)}=H_{0}+H_{e f f}^{(2)}(0)
$$


where $H_{0}$ is defined by (18). If the Schrieffer-Wolff transformation is used, then Hamiltonian (23) contains additional terms of the form [7], [8]

$$
H_{\delta}=-\frac{1}{2} \sum_{k, k^{\prime}, \sigma} \frac{V_{k^{\prime} d}}{V_{k^{\prime} d}^{*}}\left[f\left(k, k^{\prime}, U\right)-f\left(k, k^{\prime}, 0\right)\right]\left(c_{k \downarrow} c_{k^{\prime} \uparrow} d_{\uparrow}^{+} d_{\downarrow}^{+}+h . c .\right),
$$

which describe the high-energy processes, namely, the variation in the impurity level population due to the capture of two band electrons or the transition of two electrons from the d-orbital into the conduction band. The effective Hamiltonian in the KBM method does not contain such terms, because they are rapidly oscillating terms in the interaction representation and hence vanish under the averaging procedure. We neglect the terms describing the potential scattering of the electron conduction (the second term) and the electron energy renormalization on the impurity atom (the third and fourth terms) in $H_{e f f}^{(2)}(0)$ and also the Coulomb (high-energy) term in $H_{0}$ and then obtain the Hamiltonian of the Kondo model from (23) with expressions (18) and (22) taken into account:

$$
H_{e f f, H}^{(2)} \rightarrow H_{\text {Kondo }}=\sum_{k, \sigma} \varepsilon_{k} c_{k \sigma}^{+} c_{k \sigma}-\frac{1}{2} \sum_{k, k^{\prime}, \sigma}\left[f\left(k, k^{\prime}, U\right)-f\left(k, k^{\prime}, 0\right)\right]\left(c_{k \sigma}^{+} c_{k^{\prime} \bar{\sigma}} c_{d \bar{\sigma}}^{+} c_{d \sigma}-c_{k \sigma}^{+} c_{k^{\prime} \sigma} c_{d \bar{\sigma}}^{+} c_{d \bar{\sigma}}\right),
$$

where the interaction term can be expressed in spin variables of the band electrons and electrons on the d-orbital of the impurity atom [7], [8]. The KBM averaging method can also be used to consider systems under nonequilibrium conditions; for example, it can be used to study the Kondo effect in the case where the energy of an electron localized at a quantum dot is modeled by an external alternating electric field. The KBM averaging is a reliable method for constructing effective Hamiltonians for strongly correlated electron systems: high-temperature superconductors, oxide magnetics with colossal magnetoresistance, quantum dots, etc. We have illustrated the use of this method with an example of the Hubbard Hamiltonian transformation to the Hamiltonian of the t-J model and the Anderson Hamiltonian transformation to the Hamiltonian of the Kondo model. The computation algorithm is rather simple and natural. This method does not require sophisticated tricks, which are needed for choosing the specific form of the unitary operator if the canonical transformation is used. It is also unnecessary to know the eigenfunctions and eigenvalues of the Hamiltonian in the zeroth-order approximation, in contrast to the case of the perturbation theory used in systems with degenerate states. In addition, the new method is self-sufficient: it does not result in the appearance of "off- 
diagonal"terms of higher order in the perturbation parameter in the transformed Hamiltonian, which typically appear if the canonical transformation is used and are then dropped based on some plausible reasoning. This approach is very general and can be used to solve a wide range of problems in the physics of strongly correlated electron systems. In particular, it can be used to derive the effective Hamiltonian (of the t-J-model type) for the recently discovered class of high-temperature superconductors, which are iron-based layered compounds (see [9]).

\section{REFERENCES}

1. P. Fazekas, Lecture Notes on Electron Correlation and Magnetism, World Scientific, Singapore (1999).

2. Yu. A. Izyumov, Phys. Uspekhi, 40, 521-523 (1997).

3. C. L. Cleveland and R. Medina, Amer. J. Phys., 44, 44-46 (1976).

4. N. N. Bogoliubov and Y. A. Mitropolsky, Asymptotic Methods in the Theory of Non-linear Oscillations [in Russian], Nauka, Moscow (1974); English transl. prev. ed., Hindustan Publishing Corp., Delhi (1961).

5. L. L. Buishvili and M. G. Menabde, Sov. Phys. JETP, 50, 1176-1180 (1979).

6. A. P. Saiko, Phys. Solid State, 35, 20 (1993).

7. J. R. Schrieffer and P. A. Wolff, Phys. Rev., 149, 491-492 (1966).

8. P. Phillips, Advanced Solid State Physics, Westview, Boulder, Colo. (2003).

9. Yu. A. Izyumov and E. Z. Kurmaev, Phys. Uspekhi, 51, 1261-1286 (2008). 